\title{
S-1 monotherapy for previously treated non-small cell lung cancer: A retrospective analysis by age and histopathological type
}

\author{
YUKI TOMITA $^{1}$, TETSUYA OGURI ${ }^{1}$, OSAMU TAKAKUWA ${ }^{1}$, MAKOTO NAKAO ${ }^{1}$, EIJI KUNII ${ }^{1,2}$, \\ TAKEHIRO UEMURA ${ }^{1}$, HIROAKI OZASA ${ }^{1}$, MIKINORI MIYAZAKI $^{1}$, KEN MAENO $^{1}$ and SHIGEKI SATO $^{1}$ \\ ${ }^{1}$ Department of Medical Oncology and Immunology, Nagoya City University Graduate School of Medical Sciences, \\ Mizuho-cho, Mizuho-ku, Nagoya, Aichi 467-8601; ${ }^{2}$ Department of Respiratory Medicine, \\ Gifu Prefectural Tajimi Hospital, Tajimi, Gifu 507-8522, Japan
}

Received July 24, 2011; Accepted November 3, 2011

DOI: $10.3892 / \mathrm{ol} .2011 .507$

\begin{abstract}
S-1, an oral fluoropyrimidine derivative, has been approved for the treatment of non-small cell lung cancer (NSCLC) in Japan. In the present study, the efficacy and safety of S-1 monotherapy for elderly patients with previously treated NSCLC were retrospectively evaluated, and the efficacy of S-1 monotherapy was compared by histopathological type. This retrospective study included 54 patients with advanced or recurrent NSCLC who had received S-1 monotherapy following the failure of previous chemotherapy regimens at our institutes. Patient outcomes were compared based on their age and histopathological type. S-1 was administered orally, twice daily, while the duration and interval were modified according to the medical condition of each patient. The default delivery schedule, the mean number of S-1 cycles, did not differ significantly between the two age groups ( $<70$ and $\geq 70$ years). The rate of therapy discontinuation, schedule modification or dose reduction due to intolerable toxicities or patient refusal was relatively frequent in the older group (40.7 and 55.6\% for ages $<70$ and $\geq 70$ years, respectively; $\mathrm{p}=0.414$ ), and the incidence of grade 3 anemia was relatively high in the older group (3.7 and $18.5 \%$, respectively; $\mathrm{p}=0.192$ ). The response rates (13.0 and $4.8 \%$, respectively; $\mathrm{p}=0.609)$ and disease control rates $(39.1$ and $33.3 \%$, respectively; $\mathrm{p}=0.761$ ) did not differ significantly between the two age groups. According to histopathological type, the disease control rate was significantly higher in adenocarcinoma (57.9\%) compared to non-adenocarcinoma $(20.0 \%$, $\mathrm{p}=0.013$ ). Thus, $\mathrm{S}-1$ monotherapy may be equally effective and tolerated in patients $<70$ years and those $\geq 70$ years. Additionally,
\end{abstract}

Correspondence to: Dr Tetsuya Oguri, Department of Medical Oncology and Immunology, Nagoya City University Graduate School of Medical Sciences, 1 Aza-Kawasumi, Mizuho-cho, Mizuho-ku, Nagoya, Aichi 467-8601, Japan

E-mail: t-oguri@med.nagoya-cu.ac.jp

Key words: chemotherapy, elderly, lung cancer, S-1 adenocarcinoma may have a higher disease control rate than non-adenocarcinoma.

\section{Introduction}

S-1 is an oral anticancer drug that consists of tegafur, 5-chloro-2, 4-dihydroxypyridine (CDHP), and potassium oxonate in a molar ratio of 1:0.4:1. Tegafur is a prodrug of 5-fluorouracil (5-FU), and CDHP reversibly inhibits the activity of dihydropyrimidine dehydrogenase (DPD), an enzyme involved in the degradation of 5-FU. Potassium oxonate inhibits the phosphorylation of 5 -FU in the gastrointestinal tract and reduces gastrointestinal toxicities (1).

In Japan, S-1 has been approved for the treatment of non-small cell lung cancer (NSCLC), either alone or in combination with platinum compounds. However, the roles and value of S-1 monotherapy in the treatment of NSCLC have not yet been clearly defined. A phase II trial showed the efficacy of S-1 monotherapy for patients with chemotherapy-naïve NSCLC, with an overall response rate of $22.0 \%$, a median survival time (MST) of 10.2 months, and relatively high efficacy in adenocarcinoma (2). Recently, Okamoto et al demonstrated the non-inferiority of carboplatin plus S-1 relative to carboplatin plus paclitaxel in terms of overall survival (OS) for patients with advanced NSCLC (3). Therefore, the carboplatin and S-1 combination has become a therapeutic option for the first-line treatment of advanced NSCLC.

Previously, we reported the efficacy of S-1 monotherapy for NSCLC following the failure of prior chemotherapy (4). In the present study, the efficacy and safety of S-1 monotherapy for previously treated NSCLC was evaluated with respect to age $(<70$ years as the younger group and $\geq 70$ years as the older group), and the efficacy of S-1 monotherapy was compared between histopathological types (adenocarcinoma vs. non-adenocarcinoma).

\section{Patients and methods}

Patient selection. This retrospective study included patients with advanced or recurrent NSCLC who received S-1 
Table I. Patient characteristics.

\begin{tabular}{|c|c|c|c|c|c|}
\hline \multirow[t]{2}{*}{ Characteristic } & \multicolumn{2}{|c|}{ Age $<70$ years $(n=27)$} & \multicolumn{2}{|c|}{ Age $\geq 70$ years $(n=27)$} & \multirow[t]{2}{*}{$\mathrm{p}$-value $\mathrm{a}^{\mathrm{a}}$} \\
\hline & No. & $(\%)$ & No. & $(\%)$ & \\
\hline Median age, years (range) & 61 & $(41-69)$ & 73 & $(70-82)$ & \\
\hline \multicolumn{6}{|l|}{ Gender } \\
\hline Male & 17 & 63.0 & 21 & 77.8 & \multirow[t]{2}{*}{0.372} \\
\hline Female & 10 & 37.0 & 6 & 22.2 & \\
\hline \multicolumn{6}{|l|}{ Performance status (ECOG) } \\
\hline 0 & 8 & 29.6 & 8 & 29.6 & \multirow{3}{*}{1.000} \\
\hline 1 & 17 & 63.0 & 16 & 59.3 & \\
\hline 2 & 2 & 7.4 & 3 & 11.1 & \\
\hline \multicolumn{6}{|l|}{ Smoking history } \\
\hline Current or ever smoker & 16 & 59.3 & 20 & 74.1 & \multirow{3}{*}{0.512} \\
\hline Never smoked & 9 & 33.3 & 5 & 18.5 & \\
\hline Unknown & 2 & 7.4 & 2 & 7.4 & \\
\hline \multicolumn{6}{|l|}{ Histopathological type } \\
\hline Adenocarcinoma & 13 & 48.1 & 12 & 44.4 & \multirow{3}{*}{0.868} \\
\hline Squamous cell carcinoma & 10 & 37.0 & 12 & 44.4 & \\
\hline NSCLC, not specified & 4 & 14.8 & 3 & 11.1 & \\
\hline \multicolumn{6}{|l|}{ Stage of disease } \\
\hline IIIA/IIIB & 5 & 18.5 & 9 & 33.3 & \multirow[t]{2}{*}{0.352} \\
\hline IV & 22 & 81.5 & 18 & 66.7 & \\
\hline \multicolumn{6}{|l|}{ Number of prior regimens } \\
\hline 1 & 0 & 0 & 4 & 14.8 & \multirow{7}{*}{0.249} \\
\hline 2 & 8 & 29.6 & 9 & 33.3 & \\
\hline 3 & 8 & 29.6 & 6 & 22.2 & \\
\hline 4 & 5 & 18.5 & 6 & 22.2 & \\
\hline 5 & 5 & 18.5 & 2 & 7.4 & \\
\hline 6 & 0 & 0 & 0 & 0 & \\
\hline 7 & 1 & 3.7 & 0 & 0 & \\
\hline \multicolumn{6}{|l|}{ EGFR mutation/deletion } \\
\hline+ & 2 & 7.4 & 2 & 7.4 & \multirow{3}{*}{0.893} \\
\hline- & 4 & 14.8 & 6 & 22.2 & \\
\hline Unknown & 21 & 77.8 & 19 & 70.4 & \\
\hline
\end{tabular}

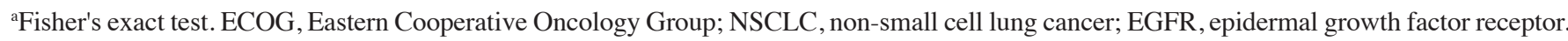

monotherapy following the failure of previous systemic chemotherapy at Nagoya City University Hospital and Gifu Prefectural Tajimi Hospital (Japan) between March 2004 and October 2010. Patients were required to fulfill the following criteria: failure of one or more regimens of systemic chemotherapy prior to S-1 administration, Eastern Cooperative Oncology Group (ECOG) performance status (PS) of 0 to 2, and adequate bone marrow, renal and liver function. Patients with other active malignancies were excluded. The medical records of eligible patients were reviewed retrospectively, and patients were observed until January 6, 2011. Approval for the study was obtained from our institutional ethics committee.

Treatment method. S-1 was administrated orally, twice daily following meals, for 4 weeks (on days 1 to 28), followed by
2 weeks of rest, every 6 weeks. The dose of S-1 was determined according to body surface area (BSA) as follows: $80 \mathrm{mg}$ /day for BSA $<1.25 \mathrm{~m}^{2}, 100 \mathrm{mg} / \mathrm{day}$ for $1.25 \mathrm{~m}^{2} \leq \mathrm{BSA}<1.50 \mathrm{~m}^{2}$, or $120 \mathrm{mg} /$ day for $\mathrm{BSA} \geq 1.50 \mathrm{~m}^{2}$. The schedule and dose for each patient were modified according to the medical condition or toxicities observed in the previous chemotherapy regimens or S-1 cycles. Administration of S-1 was continued unless there was confirmed disease progression, intolerable toxicities or patient refusal.

Evaluation and statistical analysis. Tumor responses were assessed according to the Response Evaluation Criteria in Solid Tumors. Patients who could not receive S-1 for at least 14 days were excluded from response and survival assessments. Progression-free survival (PFS) was calculated from the 


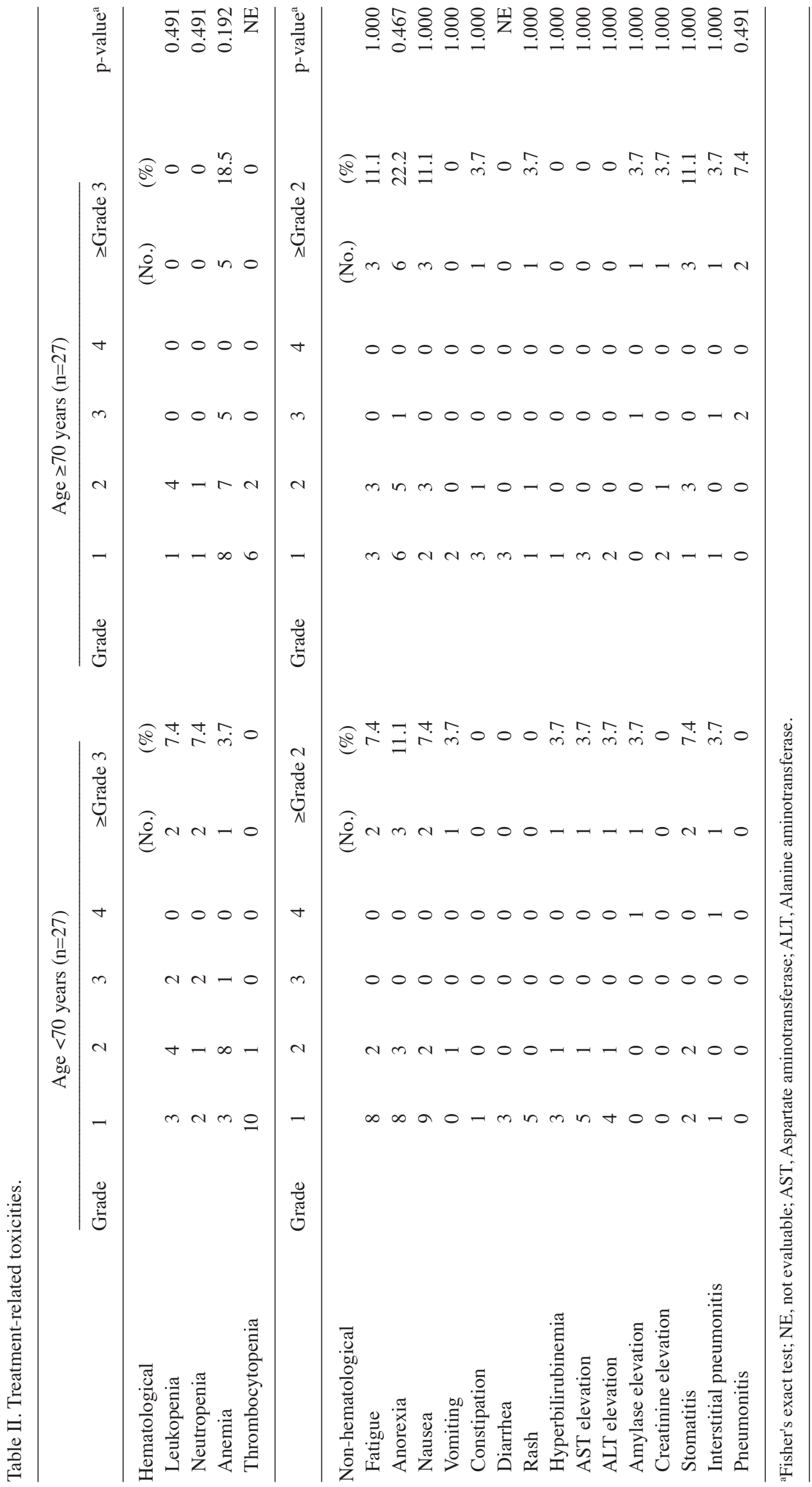


Table III. Toxicities by age group and default delivery schedule.

\begin{tabular}{llcll}
\multicolumn{1}{c}{ Age $<70$ years $(\mathrm{n}=27)$} & & Age $\geq 70$ years $(\mathrm{n}=27)$ \\
\cline { 1 - 1 } $\begin{array}{l}\text { Grade } 3 \text { hematological or } \\
\begin{array}{c}\text { Grade } 2 \text { non-hematological } \\
\text { toxicities (no.) }\end{array}\end{array}$ & $(\%)$ & $\begin{array}{c}\geq \text { Grade } 3 \text { hematological or } \\
\text { Grade } 2 \text { non-hematological } \\
\text { toxicities (no.) }\end{array}$ & $(\%)$ & p-value ${ }^{\text {a }}$
\end{tabular}

\section{Default delivery schedule \\ 4 weeks administration \\ (2 weeks of rest) \\ 3 weeks administration \\ ( 2 weeks of rest) \\ 2 weeks administration \\ (1 week of rest)}

$10 / 26$

Total

(1)

\begin{tabular}{ccccc}
$10 / 26$ & 38.5 & $13 / 23$ & 56.5 & 0.258 \\
$0 / 0$ & 0 & $1 / 1$ & 100 & $\mathrm{NE}$ \\
$1 / 1$ & 100 & $3 / 3$ & 100 & $\mathrm{NE}$ \\
$11 / 27$ & 40.7 & $17 / 27$ & 63.0 & 0.173 \\
\hline
\end{tabular}

${ }^{\mathrm{a}}$ Fisher's exact test; NE, not evaluable. date of the first day of S-1 monotherapy and the date of disease progression, and OS was calculated from the date of the first day of S-1 monotherapy and the date of mortality from any cause or the last follow-up. Treatment-related toxicities were assessed according to the Common Terminology Criteria for Adverse Events (CTCAE) version 3.0. Baseline characteristics, treatment method, response rate and toxicity were compared by Fisher's exact test. Survival time was compared using the log-rank test. The mean number of prior regimens, $\mathrm{S}-1$ cycles and subsequent regimens were compared using the two-tailed Student's t-test. Probability values of $<0.05$ were considered to be statistically significant.

\section{Results}

Patient characteristics. The clinical characteristics of the patients are shown in Table I. Of the total 54 patients, 27 patients $(50 \%)$ were $<70$ years of age (median 61 years; range 41-69 years) and 27 patients (50\%) were $\geq 70$ years of age (median 73 years; range 70-82 years). PS, smoking history, histopathological type and number of prior regimens did not differ significantly between the two age groups. The incidence of clinical stage IV was relatively high in the younger group $(81.5 \%)$ compared to the older group $(66.7 \%$, $\mathrm{p}=0.352)$. Epidermal growth factor receptor (EGFR) gene abnormality was examined only in 6 patients in the younger group ( 2 patients were harboring the deletion of exon 19 and 4 patients had wild-type EGFR), and in eight patients in the older group ( 2 patients were harboring the deletion of exon 19 and 6 patients had wild-type EGFR).

Treatment delivery. In the younger group, 26 patients $(96.3 \%)$ received the standard S-1 regimen (4 weeks administration followed by 2 weeks of rest, every 6 weeks) and 1 patient $(3.7 \%)$ received the modified S-1 regimen (2 weeks administration followed by 1 week of rest, every 3 weeks) for the first $\mathrm{S}-1$ cycle. In the older group, 23 patients $(85.2 \%)$ received the standard S-1 regimen and 4 patients $(14.8 \%)$ received modified S-1 regimens (1 patient received 3 weeks administration followed by 2 weeks of rest, every 5 weeks, and 3 patients received 2 weeks administration followed by 1 week of rest, every 3 weeks) for the first S-1 cycle. The mean number of S-1 cycles did not differ significantly between the two age groups (2.67 for the younger group and 2.48 for the older group, $\mathrm{p}=0.734)$. Therapy discontinuation, schedule modification or dose reduction due to intolerable toxicities or patient refusal was observed in 11 patients $(40.7 \%)$ in the younger group and 15 patients $(55.6 \%)$ in the older group $(\mathrm{p}=0.414)$, and all of them received the standard $\mathrm{S}-1$ regimen. At the end of the observation period, all 54 patients were no longer receiving S-1 monotherapy. Therapy discontinuation was due to disease progression (41 patients), intolerable toxicities (12 patients) and patient refusal of treatment (1 patient).

Toxicity. Treatment-related toxicities are shown in Table II. Grade 3 neutropenia was observed in 2 patients $(7.4 \%)$ in the younger group and no patients $(0 \%)$ in the older group $(\mathrm{p}=0.491)$. Grade 3 anemia was observed in 1 patient $(3.7 \%)$ in the younger group and 5 patients $(18.5 \%)$ in the older group $(p=0.192)$. Grade 4 hematological toxicity was not observed in either of the groups. The incidence of grade 2 to 4 non-hematological toxicities did not differ significantly between the two age groups, and the incidence of grade 3 or 4 non-hematological toxicities was very low. Toxicities by default delivery schedule did not differ significantly between the two age groups (Table III). No death related to S-1 monotherapy occurred.

Efficacy. Of the total 54 patients, treatment response and survival were not assessed in 10 patients who were unable to receive S-1 for at least 14 days. Among the 44 remaining patients, the response rates $(13.0 \%$ for the younger group and $4.8 \%$ for the older group, $\mathrm{p}=0.609)$ and the disease control rates (39.1 and $33.3 \%, \mathrm{p}=0.761$ ) did not differ significantly between the two age groups (Table IV). The median PFS was 3.5 months in the younger group and 2.5 months in the older group. The MST was 15.1 months in the younger group and 6.0 months in the older group. Neither PFS nor OS differed significantly between the two age groups (PFS, $\mathrm{p}=0.115$; OS, $\mathrm{p}=0.187$ ). According to 
Table IV. Response and survival by age group.

\begin{tabular}{rrrrrr}
\multicolumn{2}{c}{ Age $<70$ years $(\mathrm{n}=23)$} & & \multicolumn{2}{c}{ Age $\geq 70$ years $(\mathrm{n}=21)$} & $\mathrm{p}$-value \\
\cline { 1 - 1 } No. & $(\%)$ & & No. & $(\%)$ & \\
& & & & \\
0 & 0.0 & & 0.0 & - \\
3 & 13.0 & 1 & 4.8 & - \\
6 & 26.1 & 6 & 28.6 & - \\
14 & 60.9 & 14 & 66.7 & - \\
3 & 13.0 & 1 & 4.8 & $0.609^{\mathrm{a}}$ \\
9 & 39.1 & 7 & 33.3 & $0.761^{\mathrm{a}}$
\end{tabular}

Median PFS (months)

MST (months)

Mean no. prior regimens (range)

Mean no. subsequent regimens (range)
3.5

15.1

$3.4(2-7)$

$1.3(0-4)$
2.5

6.0

$2.6(1-5)$

$0.6(0-3)$ $0.061^{\mathrm{b}}$ $0.029^{\mathrm{b}}$

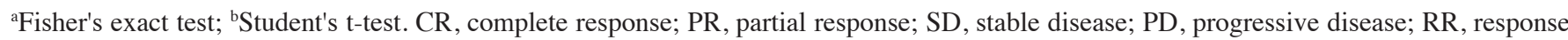
rate; DCR, disease control rate; PFS, progression-free survival; MST, median survival time.

Table V. Response and survival by histopathological type.

\begin{tabular}{crrrrr}
\multicolumn{2}{c}{ Adenocarcinoma $(\mathrm{n}=19)$} & & \multicolumn{2}{c}{ Non-adenocarcinoma $(\mathrm{n}=25)$} & p-value \\
\cline { 1 - 1 } No. & $(\%)$ & & No. & $(\%)$ & \\
& & & & & \\
0 & 0.0 & & & 0.0 & - \\
3 & 15.8 & & & 4.0 & - \\
8 & 42.1 & & 16.0 & - \\
8 & 42.1 & 20 & 80.0 & - \\
3 & 15.8 & 1 & 4.0 & $0.300^{\mathrm{a}}$ \\
11 & 57.9 & 5 & 20.0 & $0.013^{\mathrm{a}}$
\end{tabular}

Median PFS (months)

MST (months)

Performance status (ECOG)

0

1

2

Stage of disease

IIIA
IIIB
IV

Mean age, years (range)

$64.5(41-82)$

52.6

42.

42.1

6

15

2.4

6.1

4.2

15.7

8

1

0

3

16

$\begin{array}{rr}0.0 & 2 \\ 15.8 & 6 \\ 84.2 & 17\end{array}$

24.0

60.0

$0.132^{\mathrm{a}}$

16.0

8.0

24.0

$0.461^{\mathrm{a}}$

68.0 rate; DCR, disease control rate; PFS, progression-free survival; MST, median survival time; ECOG, Eastern Cooperative Oncology Group. 
histolopathological type (Table V), the response rates did not differ significantly between adenocarcinoma (15.8\%) and nonadenocarcinoma $(4.0 \%, \mathrm{p}=0.300)$, while the disease control rate was significantly higher for adenocarcinoma $(57.9 \%)$ than for non-adenocarcinoma $(20.0 \%, \mathrm{p}=0.013)$. Median PFS was 4.2 months for adenocarcinoma and 2.4 months for nonadenocarcinoma. MST was 15.7 months for adenocarcinoma and 6.1 months for non-adenocarcinoma. Neither PFS nor OS differed significantly between the two histopathological groups (PFS, $\mathrm{p}=0.068$; OS, $\mathrm{p}=0.684$ ).

\section{Discussion}

In the present study, the efficacy and safety of S-1 monotherapy were retrospectively analyzed in patients with advanced or recurrent NSCLC who had previously received one or more regimens of chemotherapy. Of the total 54 patients, $49(90.7 \%$; 26 in the younger group and 23 in the older group) received the standard S-1 regimen for the first S-1 cycle. Of these patients, the incidence of grade 3 hematological toxicities or grade 2-4 non-hematological toxicities was $38.5 \%$ in the younger group and $56.5 \%$ in the older group $(\mathrm{p}=0.258)$. Additionally, therapy discontinuation, schedule modification or dose reduction due to intolerable toxicities or patient refusal was observed in $11(42.3 \%)$ in the younger group and $15(65.2 \%)$ in the older group $(\mathrm{p}=0.154)$. These findings suggest a relatively increased susceptibility to toxicities in older patients who received the standard regimen. Recently, a modified S-1 regimen (2 weeks administration followed by 1 week of rest, every 3 weeks) has been reported to be effective and well tolerated in elderly patients with NSCLC (5). Therefore, modified S-1 regimens for elderly patients should be further investigated.

The response rates, disease control rates, PFS and OS did not differ significantly between the two age groups. Furthermore, the response rates did not differ significantly between the two histopathological groups, whereas the disease control rate was significantly higher for adenocarcinoma $(57.9 \%)$ than for non-adenocarcinoma patients $(20.0 \%$, $\mathrm{p}=0.013$ ). Although neither PFS nor OS differed significantly between the two histopathological groups, the median PFS period was longer in adenocarcinoma (4.2 months) compared to non-adenocarcinoma (2.4 months).

The antitumor activity of S-1 is primarily derived from its antagonistic activity against thymidylate synthase (TS), which has been reported to have higher expression levels in squamous cell carcinoma than in non-squamous cell carcinoma $(1,6)$. A number of mechanisms, including a high TS expression level, have been reported in association with resistance to 5-FU and its derivatives (7-11). Clinical investigations suggest a survival advantage for TS-targeting agents in patients with non-squamous cell carcinoma (12-13). The response advantage of S-1 monotherapy for adenocarcinoma in the present study may be due to the same mechanisms.
In conclusion, S-1 monotherapy appears to be equally effective and tolerated in younger and older patients with previously treated NSCLC, while the incidence of therapy discontinuation, schedule modification or dose reduction due to intolerable toxicities or patient refusal was relatively high in older patients, particularly in those who received the standard S-1 regimen. In addition, while prolongation of PFS or OS was not confirmed, S-1 monotherapy may provide a higher disease control rate for adenocarcinoma than for non-adenocarcinoma. To clarify these findings, further clinical, molecular biological and biopharmaceutical investigations are required.

\section{References}

1. Shirasaka T, Nakano K, Takechi T, et al: Antitumor activity of $1 \mathrm{M}$ tegafur-0.4 M 5-chloro-2,4-dihydroxypyridine-1 M potassium oxonate (S-1) against human colon carcinoma orthotopically implanted into nude rats. Cancer Res 56: 2602-2606, 1996.

2. Kawahara M, Furuse K, Segawa Y, et al: Phase II study of S-1, a novel oral fluorouracil, in advanced non-small-cell lung cancer. Br J Cancer 85: 939-943, 2001.

3. Okamoto I, Yoshioka H, Morita S, et al: Phase III trial comparing oral S-1 plus carboplatin with paclitaxel plus carboplatin in chemotherapy-naïve patients with advanced non-small-cell lung cancer: results of a West Japan Oncology Group study. J Clin Oncol 28: 5240-5246, 2010.

4. Takakuwa O, Oguri T, Maeno K, et al: Efficacy of S-1 monotherapy for non-small cell lung cancer after the failure of two or more prior chemotherapy regimens. Oncol Lett 1: 147-150, 2010.

5. Nishiyama O, Taniguchi $\mathrm{H}$, Kondoh Y, et al: Phase II study of S-1 monotherapy as a first-line treatment for elderly patients with advanced nonsmall-cell lung cancer: the Central Japan Lung Study Group trial 0404. Anticancer Drugs 22: 811-816, 2011.

6. Ceppi P, Volante M, Saviozzi S, et al: Squamous cell carcinoma of the lung compared with other histotypes shows higher messenger RNA and protein levels for thymidylate synthase. Cancer 107: 1589-1596, 2006.

7. Oguri T, Achiwa H, Bessho Y, et al: The role of thymidylate synthase and dihydropyrimidine dehydrogenase in resistance to 5-fluorouracil in human lung cancer cells. Lung Cancer 49: 345-351, 2005.

8. Oguri T, Bessho Y, Achiwa H, et al: MRP8/ABCC11 directly confers resistance to 5 fluorouracil. Mol Cancer Ther 6: 122-127, 2007.

9. Giovannetti E, Mey V, Nannizzi S, et al: Cellular and pharmacogenetics foundation of synergistic interaction of pemetrexed and gemcitabine in human non-small-cell lung cancer cells. Mol Pharmacol 68: 110-118, 2005.

10. Ozasa H, Oguri T, Uemura T, et al: Significance of thymidylate synthase for resistance to pemetrexed in lung cancer. Cancer Sci 101: 161-166, 2010.

11. Gomez HL, Santillana SL, Vallejos CS, et al: A phase II trial of pemetrexed in advanced breast cancer: clinical response and association with molecular target expression. Clin Cancer Res 12: 832-838, 2006.

12. Scagliotti GV, Parikh P, von Pawel J, et al: Phase III study comparing cisplatin plus gemcitabine with cisplatin plus pemetrexed in chemotherapy-naive patients with advanced-stage non-small-cell lung cancer. J Clin Oncol 26: 3543-3551, 2008.

13. Scagliotti G, Hanna N, Fossella F, et al: The differential efficacy of pemetrexed according to NSCLC histology: a review of two phase III studies. Oncologist 14: 253-263, 2009. 